\title{
't Hooft Loop in SU(2) Yang-Mills Theory
}

\author{
Philippe de Forcrand, ${ }^{1,2}$ Massimo D'Elia, ${ }^{3}$ and Michele Pepe ${ }^{1}$ \\ ${ }^{1}$ Institut für Theoretische Physik, ETH Hönggerberg, CH-8092 Zürich, Switzerland \\ ${ }^{2}$ CERN, Theory Division, CH-1211 Genève 23, Switzerland \\ ${ }^{3}$ Dipartimento di Fisica dell'Università and I.N.F.N., I-56127, Pisa, Italy
}

(Received 25 July 2000)

\begin{abstract}
We study the behavior of the spatial and temporal 't Hooft loop at zero and finite temperature in the 4D SU(2) Yang-Mills theory, using a new numerical method. In the deconfined phase $T>T_{c}$, the spatial 't Hooft loop exhibits a dual string tension, which vanishes at $T_{c}$ with a $3 \mathrm{D}$ Ising-like critical exponent.
\end{abstract}

DOI: 10.1103/PhysRevLett.86.1438

The 4D SU(2) Yang-Mills theory undergoes a transition between a cold confined phase and a hot deconfined phase at a critical temperature $T_{c}$. An order parameter widely used to characterize this transition is the Polyakov loop. It develops a nonvanishing expectation value in the deconfined phase. However, the corresponding operator creates a single fundamental static color source, which does not belong to the physical Hilbert space of the theory; it cannot be defined at zero temperature; and it is afflicted by ultraviolet divergences in the continuum limit [1,2]. A long time ago it was proposed [3] to consider instead the 't Hooft loop operator as an order parameter to characterize this transition. It is the purpose of this paper to study this order parameter. The 't Hooft loop, $\tilde{W}(C)$, is an operator associated with a given closed contour $C$ and is defined in the continuum $\mathrm{SU}(N)$ theory by the following equal-time commutation relations [3]:

$$
\begin{gathered}
{\left[W(C), W\left(C^{\prime}\right)\right]=\left[\tilde{W}(C), \tilde{W}\left(C^{\prime}\right)\right]=0,} \\
\tilde{W}^{\dagger}(C) W\left(C^{\prime}\right) \tilde{W}(C)=e^{i \frac{2 \pi}{N} n_{C C^{\prime}}} W\left(C^{\prime}\right),
\end{gathered}
$$

where $W\left(C^{\prime}\right)$ is the Wilson loop associated with the closed contour $C^{\prime}$ and $n_{C C^{\prime}}$ is the linking number of $C$ and $C^{\prime}$. Just like the Wilson loop creates an elementary electric flux along $C^{\prime}$, the 't Hooft loop creates an elementary magnetic flux along the closed path $C$ affecting any Wilson loop "pierced" by $C$. In that sense, the two types of loop are dual to each other. At zero temperature, it has been shown [3-5] that also the 't Hooft loop behavior is dual to that of the Wilson loop: in the absence of massless excitations, an area law behavior for one implies a perimeter law for the other, and vice versa. Hence, at $T=0$ the 't Hooft loop obeys a perimeter law.

Several analytical $[2,6]$ and numerical [7-9] studies have been carried out in order to investigate this issue of duality at finite temperature. At $T>0$, the Lorentz symmetry is broken, so spatial and temporal loops can have different behaviors. Because the spatial string tension persists also above $T_{c}$ for the Wilson loop, temporal 't Hooft loops are expected to show a perimeter law in both phases; spatial 't Hooft loops are expected to obey a perimeter law in the confined phase and an area law_defining a dual
PACS numbers: 12.38.Gc, 11.15.Ha, 12.38.Aw

string tension (strictly speaking it is an action density) - in the deconfined phase.

On the lattice the 't Hooft loop is defined as follows. Let us consider the $\mathrm{SU}(2)$ lattice gauge theory with the usual Wilson plaquette action,

$$
S(\beta)=\beta \sum_{P}\left(1-\frac{1}{2} \operatorname{Tr}\left(U_{P}\right)\right),
$$

where the sum extends over all the plaquettes $P$, and $U_{P}$ is the path-ordered product of the links around $P$. Starting from $S(\beta)$, one defines the partition function

$$
Z(\beta)=\int[d U] \exp (-S(\beta)) .
$$

Let us now switch on "by hand" an elementary magnetic flux along a closed contour $C$ defined on the dual lattice. To create this magnetic flux, we have to multiply $U_{P}$ by a nontrivial element of the center group for the plaquettes $P$ dual to a given surface $S$ supported by $C[10,11]$. For the $\mathrm{SU}(2)$ gauge group, this means flipping the sign of the coupling, since the only nontrivial element of the center $Z_{2}$ is -1 . Call $\mathcal{P}(S)$ the set of plaquettes whose coupling is flipped $\beta \rightarrow-\beta$. Then the action of the system where an elementary flux along a closed contour $C$ has been switched on is given, up to an additive constant, by

$$
S_{S}(\beta)=-\frac{1}{2} \beta\left(\sum_{P \notin \mathcal{P}(S)} \operatorname{Tr}\left(U_{P}\right)-\sum_{P \in \mathcal{P}(S)} \operatorname{Tr}\left(U_{P}\right)\right),
$$

and the partition function is

$$
Z_{C}(\beta)=\int[d U] \exp \left(-S_{S}(\beta)\right) .
$$

$Z_{C}(\beta)$ does not depend on the particular chosen surface $S$, since different choices are related by a change of integration variables. The simplest choice for $S$ is the minimal surface spanning $C$. Thus, if $C$ is an $R_{x} \times R_{y}$ rectangle in the $(x, y)$ plane (spatial 't Hooft loop), one flips the coupling of the $(z, t)$ plaquettes dual to the plaquettes belonging to the rectangular area.

The 't Hooft loop expectation value is related to the free energy cost needed to create the magnetic flux along the contour $C$ and is given by 


$$
\langle\tilde{W}(C)\rangle=Z_{C}(\beta) / Z(\beta) .
$$

This expression can be rewritten in the form

$$
\langle\tilde{W}(C)\rangle=\left\langle\exp \left(-\beta \sum_{P \in \mathcal{P}(S)} \operatorname{Tr}\left(U_{P}\right)\right)\right\rangle,
$$

with the average taken with the standard Wilson action. In this form, the difficulty of measuring the 't Hooft loop becomes clear: the observable is exponentially suppressed on typical configurations of the statistical ensemble and gets a significant contribution only from configurations having an extremely small statistical weight. Therefore the numerical evaluation of $\langle\tilde{W}(C)\rangle$ represents a difficult sampling problem, increasingly so with the loop size.

Recently, the 't Hooft loop, or special cases of it, has been studied numerically on the lattice. In $[7,8]$ the sampling problem was overcome by using a multihistogram method, where one performs several different simulations in which the coupling associated with the plaquettes in $\mathcal{P}(S)$ is gradually changed from $\beta$ to $-\beta$. In [9], instead, the derivative $d / d \beta \ln \langle\tilde{W}(C)\rangle$ has been determined, which is a much simpler numerical task.

In this Letter we report on a similar numerical study where, by measuring spatial and temporal 't Hooft loops at zero and finite temperature, we confirm the role of the 't Hooft loop as a dual order parameter for confinement. We adopt a new numerical method, rewriting the ratio $Z_{C}(\beta) / Z(\beta)$ as a product of intermediate ratios, each easily measurable. We establish the perimeter law behavior of the 't Hooft loop at zero temperature. We measure the free energy of two center monopoles as a function of their separation: as expected from duality, temporal 't Hooft loops show screening behavior at all temperatures, while spatial 't Hooft loops exhibit a dual string tension above $T_{c}$. Moreover, this dual string tension vanishes at $T_{c}$ with a critical exponent $\nu$ very close to that of the 3D Ising model, consistent with universality.

The numerical technique.-We now describe our numerical method to measure the expectation value of the 't Hooft operator. Because the relevant contributions to $Z_{C}(\beta)$ and to $Z(\beta)$ come from regions of the phase space with very poor overlap, a direct evaluation of (7) and (8) by a single Monte Carlo simulation is not a reliable way to compute $\langle\tilde{W}(C)\rangle$. It is necessary to consider a sequence of intermediate partition functions which interpolate between $Z(\beta)$ and $Z_{C}(\beta)$. The approach used in $[7,8]$ consists of interpolating in the coupling of the flipped plaquettes in $\mathcal{P}(S)$, from $\beta$ to $-\beta$. We interpolate instead in the number of flipped plaquettes and rewrite $Z_{C}(\beta) / Z(\beta)$ as the product of ratios of partition functions where the number of plaquettes with flipped coupling is progressively reduced. $N$ being the number of plaquettes in $\mathcal{P}(S)$, we use the identity

$$
\frac{Z_{C}(\beta)}{Z(\beta)}=\frac{Z_{N}(\beta)}{Z_{N-1}(\beta)} \frac{Z_{N-1}(\beta)}{Z_{N-2}(\beta)} \cdots \frac{Z_{1}(\beta)}{Z_{0}(\beta)},
$$

where $Z_{k}(\beta), k=0, \ldots, N\left(Z_{N} \equiv Z_{C}\right.$ and $\left.Z_{0} \equiv Z\right)$ is the partition function of the system where only the first $k$ plaquettes in $\mathcal{P}(S)$ have flipped coupling. Every ratio $Z_{k} / Z_{k-1}$ can now be computed efficiently by a single Monte Carlo simulation, due to the good overlap of the relevant phase space in the two partition functions. Furthermore, in the practical implementation, it is useful to reexpress $Z_{k}(\beta) / Z_{k-1}(\beta)$ as a ratio of expectation values

$$
\frac{Z_{k}(\beta)}{Z_{k-1}(\beta)}=\frac{\left\langle\exp \left[-\frac{1}{2} \beta \operatorname{Tr}\left(U_{P_{k}}\right)\right]\right\rangle_{k}}{\left\langle\exp \left[+\frac{1}{2} \beta \operatorname{Tr}\left(U_{P_{k}}\right)\right]\right\rangle_{k}} .
$$

The averages $\langle\cdot\rangle_{k}$ are computed with respect to an action where the $k$ th plaquette $U_{P_{k}}$ of $\mathcal{P}(S)$ has zero coupling, the first $(k-1)$ plaquettes have coupling $-\beta$, and the remaining ones coupling $+\beta$. The benefits over simply computing $\left\langle\exp \left[-\beta \operatorname{Tr}\left(U_{P_{k}}\right)\right]\right\rangle$ using the distribution corresponding to $Z_{k-1}(\beta)$ are twofold. (i) Using the intermediate weight, $\langle\cdot\rangle_{k}$, allows a much better sampling for both $\left\langle\exp \left[-\frac{1}{2} \beta \operatorname{Tr}\left(U_{P_{k}}\right)\right]\right\rangle_{k}$ and $\left\langle\exp \left[+\frac{1}{2} \beta \operatorname{Tr}\left(U_{P_{k}}\right)\right]\right\rangle_{k}$, with reduced errors. (ii) Since the quantity $Z_{k}(\beta) / Z_{k-1}(\beta)$ refers to a single plaquette of the lattice, it is very useful to perform a partial integration after each updating sweep of the whole lattice and measure $\exp \left[+\frac{\beta}{2} \operatorname{Tr}\left(U_{P_{k}}\right)\right]$ and $\exp \left[-\frac{\beta}{2} \operatorname{Tr}\left(U_{P_{k}}\right)\right]$ several times while updating only the four links belonging to plaquette $P_{k}$. Since this plaquette has zero coupling, these four links are decoupled from each other. Therefore each link can be updated independently $N_{\text {hit }}$ times and the different copies of each link can be combined to obtain $N_{\text {hit }}^{4}$ measurements of $\exp \left[ \pm \frac{\beta}{2} \operatorname{Tr}\left(U_{P_{k}}\right)\right]$. Although these measurements are correlated, the variance reduction is important.

The statistical error on ratio (10) must be evaluated with care, since the two averages are computed from the same sample of configurations. We use a jackknife analysis. Then, after each $Z_{k}(\beta) / Z_{k-1}(\beta)$ has been computed, the 't Hooft loop expectation value is evaluated according to Eq. (9). The final statistical error is obtained by standard error propagation since each $Z_{k}(\beta) / Z_{k-1}(\beta)$ comes from an independent Monte Carlo simulation.

An advantage of our method over the multihistogram technique $[7,8]$ is that the products in (9) give us information on smaller 't Hooft loops for free; moreover, the error analysis is simpler and less delicate than in a multihistogram analysis.

Results.-We focus on the free energy $F(R)$ of a pair of static center monopoles as a function of their separation $R$. It can be obtained as $\lim _{R_{t} \rightarrow \infty}-\operatorname{Ln}\left[\left\langle\tilde{W}\left(R, R_{t}\right)\right\rangle\right] / R_{t}$ by taking elongated $R \times R_{t}$ rectangular loops, in the same way as one extracts the static potential between two chromoelectric charges. We take $R_{t}$ as large as possible, i.e., equal to the lattice size $L$. This is analogous to measuring the correlation of two Polyakov loops and is the correct approach at finite temperature. Therefore we must flip the coupling of $R \times L$ plaquettes. We do this by scanning first the $R_{t}$, then the $R$ direction. With this ordering, the 


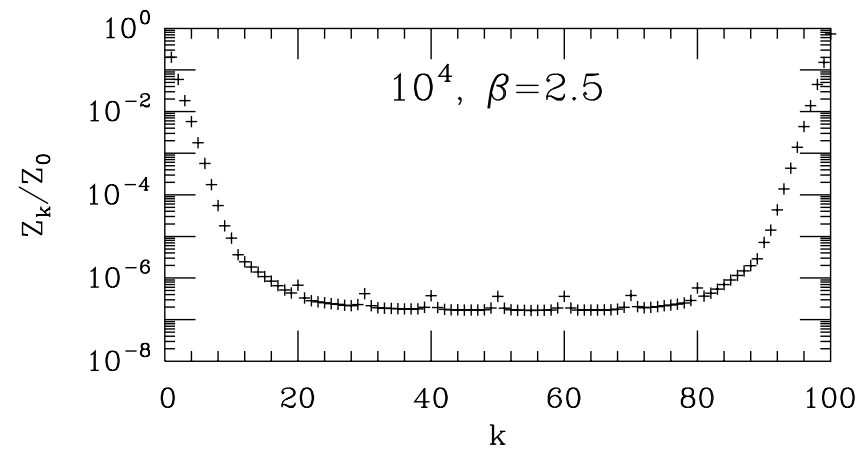

FIG. 1. Partition function $Z_{k} / Z_{0}$ versus $k$. Incrementing $k$ always increase by 1 the area of the 't Hooft loop, but changes its perimeter only by $\mp 2$ if $k=0$ or $1 \bmod (10)$, respectively. $Z_{k}$ is clearly sensitive to changes of perimeter but not of area.

intermediate partition functions $Z_{L}, Z_{2 L}, \ldots, Z_{R \times L}$ in (9) provide us with the free energy at separations $1,2, \ldots, R$, respectively. The final ratio $Z_{L \times L} / Z_{0}$ gives the free energy of a center vortex as computed in [7]. Our lattice sizes range from $10^{3} \times 2$ to $20^{3} \times 10$, at couplings $\beta=\frac{4}{g^{2}}$ from 2.3 to 2.8 , for which the lattice spacing varies by a factor $\sim 5$. We perform 5-10 000 (multihit) measurements of each ratio (10).

At zero temperature the 't Hooft loop is expected to obey a perimeter law: $Z_{k} / Z_{0} \propto e^{-c \tilde{P}_{k}}$, where $\tilde{P}_{k}$ is the length of the contour $C_{k}$. For the sequence of flipped plaquettes defined above, $\tilde{P}_{k}=2 L$ if $\bmod (k, L)=0,(2 L+$ 2) otherwise, unless $k<L$ or $k>L(L-1)$. $Z_{k}$ should therefore center around two values only. This is exactly what appears in Fig. 1, on a $10^{4}$ lattice. The free energy is clearly insensitive to changes in the 't Hooft loop area. This is also confirmed by a direct measurement of Creutz ratios $\chi(R, R)$, which estimate the force at distance $R$ between the two magnetic charges and quickly drop to zero as $R$ increases. As with the Wilson loop, the coefficient $c$ of the perimeter term is affected by UV divergences. We verified that it increases as the lattice spacing is reduced.

The free energy $F(r)$ can be fitted by a Yukawa form $\frac{e^{-m r}}{r}$, up to an irrelevant additive constant. However, the screening mass $m$ is rather large, so that the signal quickly dies out. Moreover, consistency of data at different values of the lattice spacing is only fair. This is presumably caused by short-distance lattice distortions of the Yukawa potential, which could be explicitly taken into account. In any case, Fig. 2 shows the free energy as a function of the magnetic charge separation. The curve corresponds to a screening mass of $2 \mathrm{GeV}$. It makes sense to match this mass with the lightest gluonic excitation, the scalar glueball $(\sim 1.65 \mathrm{GeV})$, as attempted in [8,9]; but this issue awaits a more extensive numerical study.

At finite temperature we must distinguish between spatial and temporal 't Hooft loops. In the case of electric charges, the spatial string tension persists above $T_{c}$, while the correlation of timelike Polyakov loops develops a disconnected part showing saturation of the static potential.

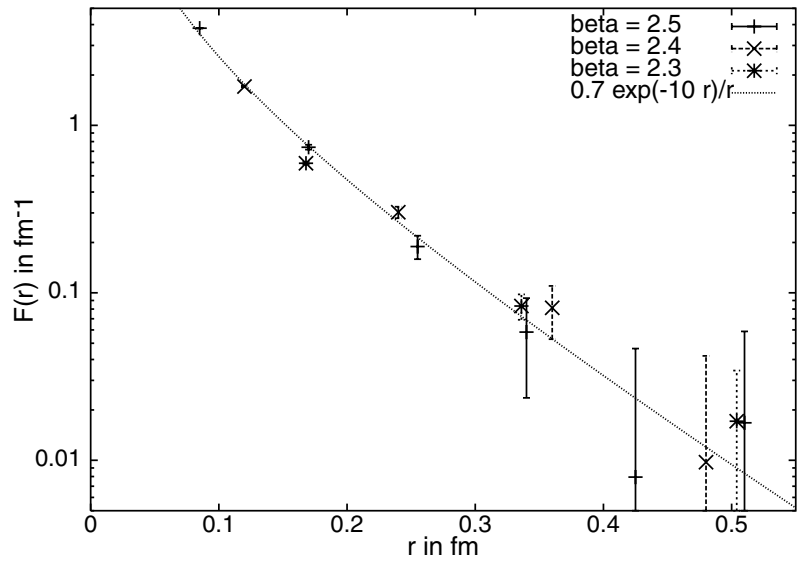

FIG. 2. Free energy of a static pair of center monopoles as a function of their separation, at temperature $T<T_{c}$. The scaling behavior of the data is mediocre. The curve shows a Yukawa potential with screening mass $2 \mathrm{GeV}$.

Here the temporal 't Hooft loop should show screening, while the spatial 't Hooft loop should obey an area law. It is clear that the latter will cost more in free energy, since the center vortex created by each flipped $(z, t)$ plaquette can spread only over a limited time extent $T^{-1}$.

Indeed, this is precisely what we observe. Figure 3 shows the same Yukawa form for a temporal 't Hooft loop at all temperatures. The only effect of temperature is to increase the screening mass. The fitting coefficient of the Yukawa potential is smaller than the short-distance perturbative prediction $1 / 4 \pi(2 \pi / g)^{2}$ [12] but grows towards this value at higher $\beta$. An attempt to fit the data with the ansatz $F_{0}+c \frac{e^{-m r}}{r}+\sigma r$, including a linear term, gives a dual string tension $\sigma$ consistent with zero. In contrast, this linear term is required to obtain an acceptable fit above $T_{c}$ for the spatial 't Hooft loop: a dual string tension appears. As a practical consequence, the screening mass becomes yet harder to determine. It seems little affected by temperature, as Fig. 4 (top) shows, unlike the temporal

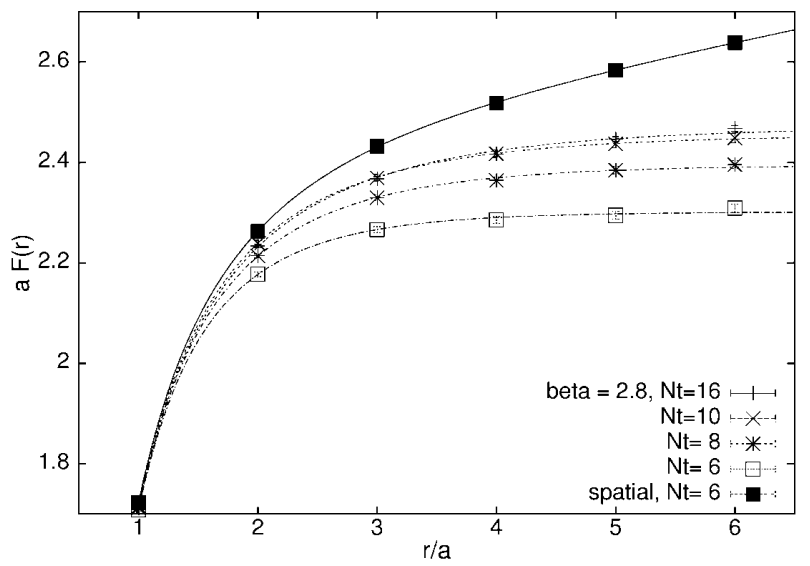

FIG. 3. Free energy of a static pair of center monopoles as a function of their separation at temperature $T>T_{c}$. The spatial 't Hooft loop shows a dual string tension; the temporal 't Hooft loop shows a screening mass which increases with $T$. 


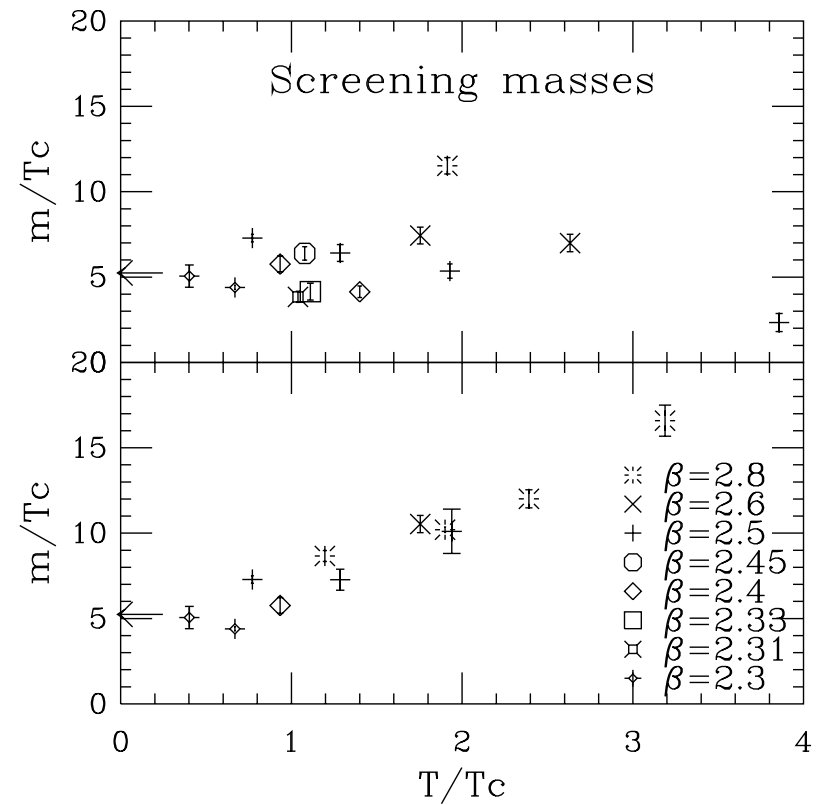

FIG. 4. Screening mass as a function of temperature, both in units of $T_{c}$, as extracted from spatial (top) or temporal (bottom) 't Hooft loops. Below $T_{c}$ both coincide. The arrow gives the mass of the scalar glueball at $T=0$.

screening mass (bottom) which rises more or less linearly with $T$, much like the glueball excitation which it presumably represents [13]. Precise quantitative statements about these dependences on $T$ require a more accurate numerical investigation.

The dual string tension $\sigma$ depends on temperature and must vanish at $T_{c}$. Figure 5 shows that it does so as $\sigma \propto\left(\frac{T-T_{c}}{T_{c}}\right)^{2 \nu}$. The critical exponent $\nu$, associated with the correlation length $\xi=\sigma^{-1 / 2}$, comes out very close to that of the $3 \mathrm{D}$ Ising model: $0.66(3)$ vs $\approx 0.63$. Indeed this should be expected since both models are in the same universality class, although the Ising exponent is typically extracted from the divergence of $\xi$ in the symmetric (confined) phase. This dual string tension can then be taken as order parameter for the restoration of the (magnetic) $Z_{N}$ symmetry, corresponding to deconfinement $[2,14]$.

Figure 5 also shows high $T$, perturbative results. Notice that a spatial $(x, y)$ 't Hooft loop of maximal size $L \times L$ introduces a flipped plaquette in every $(z, t)$ plane, which is equivalent to enforcing twisted boundary conditions in the $(z, t)$ directions, thus creating a $Z_{N}$ interface with tension $(\sigma T)$. This interface tension has been calculated to two loops [6]. Taking the running coupling $g(T)$ from [15], one obtains the two curves in Fig. 5 for the leading and next order. The numerical data lie in between.

In summary, by using a dual observable, we have measured a dual string (or interface) tension in the deconfined phase. The corresponding correlation length diverges at $T_{c}$ with the 3D Ising critical exponent $\nu$ as expected from universality. An extension to $\mathrm{SU}(3)$ is straightforward. In that case, since the transition is first order, the dual string

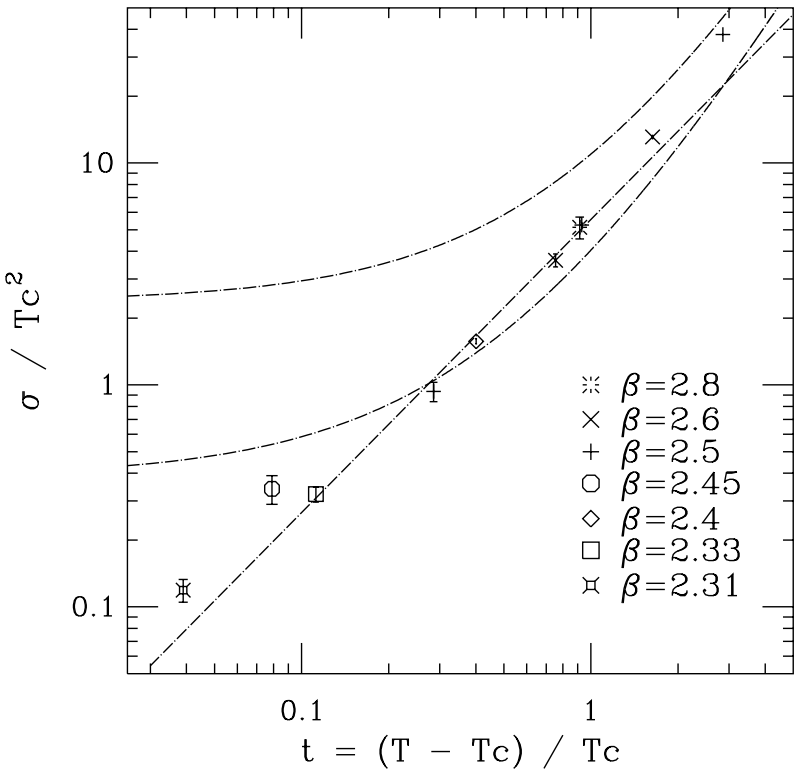

FIG. 5. Dual string tension, in units of $T_{c}^{2}$, as a function of the reduced temperature $t$. The straight line is a power law fit to $t<1$. The fitted exponent is 1.32(6), to be compared with $2 \nu \approx 1.26$ for the $3 \mathrm{D}$ Ising model. The curves show the perturbative result, to leading (upper) and next (lower) order.

tension will persist all the way to $T_{c}$. Its value at $T_{c}$ has been the object of many studies [16]. Finally, it would be very desirable to measure more precisely the screening mass and to clarify its physical origin.

We thank C. Alexandrou, P. Butera, L. Del Debbio, A. Di Giacomo, C. Korthals-Altes, M. Laine, and O. Philipsen for many helpful discussions and acknowledge communication with T. Kovács, T. Tomboulis, and C. Rebbi.

[1] A. Smilga, Ann. Phys. (N.Y.) 234, 1 (1994).

[2] C. Korthals-Altes, A. Kovner, and M. Stephanov, Phys. Lett. B 469, 205-212 (1999).

[3] G. 't Hooft, Nucl. Phys. B138, 1 (1978).

[4] E. Tomboulis, Phys. Rev. D 23, 2371 (1981).

[5] S. Samuel, Nucl. Phys. B214, 532 (1983).

[6] T. Bhattacharya et al., Nucl. Phys. B383, 497 (1992).

[7] T. G. Kovács and E. T. Tomboulis, hep-lat/0002004.

[8] Ch. Hoelbling, C. Rebbi, and V.A. Rubakov, hep-lat/ 9809113; hep-lat/9909023; hep-lat/0003010.

[9] L. Del Debbio, A. Di Giacomo, and B. Lucini, hep-lat/ 0006028 .

[10] A. Ukawa, P. Windey, and A. H. Guth, Phys. Rev. D 21, 1013 (1980).

[11] M. Srednicki and L. Susskind, Nucl. Phys. B179, 239 (1981).

[12] M. N. Chernodub et al., hep-th/0003138; hep-th/0007135.

[13] S. Datta and S. Gupta, hep-lat/9906023.

[14] C. Korthals-Altes and A. Kovner, hep-ph/0004052.

[15] K. Kajantie et al., Nucl. Phys. B503, 357 (1997), Eq. (2.24).

[16] See, e.g., Y. Iwasaki et al., Phys. Rev. D 49, 3540 (1994). 